\title{
Aplicando Internet das Coisas na Educação: Tecnologia, Cenários e Projeções
}

\author{
Rafael de Amorim Silva ${ }^{1}$, João Gabriel G.V. Nova ${ }^{1}$, Rubem F.S. Vasconcelos ${ }^{1}$, Ivo \\ Calado A.A.R. Calado ${ }^{2}$, Kalinka R.L.J.C. Branco ${ }^{3}$, Rosana T.V. Braga ${ }^{3}$ \\ ${ }^{1}$ Instituto de Computação - Universidade Federal de Alagoas (UFAL) \\ Caixa Postal 61 - 57.300-970 - Maceió - AL - Brasil \\ ${ }^{2}$ Instituto de Computação - Instituto Federal de Alagoas (IFAL) \\ Palmeira dos Índios - AL - Brasil \\ ${ }^{3}$ Instituto de Ciências Matemáticas e Computação - Universidade de São Paulo (USP) \\ São Carlos - SP - Brasil \\ \{rafael, jgg, rfsv\}@ic.ufal.br, ivocalado@gmail.com, \{rtvb, \\ kalinka\}@icmc.usp.br
}

\begin{abstract}
In the last years, industry, government and research institutions have made efforts to develop a technology that connects physical objects able to interchange information and to sense details of their own environment, enabling strategic data for a better decision making. Such technology is called Internet of Things (IoT), a distinct network established among heterogeneous objects to provide services and applications for several domain areas. This network encompasses features and operations that are different from traditional networks, being a potential technology for usage in the apprentice learning process in future classrooms. This paper discusses the usage of this network in the educational context, identifying killer applications that improve the efficiency of teaching and enable a major engagement and interest of students and teachers.

Resumo. Nos últimos anos, a indústria, o governo e as instituições de pesquisa têm unido esforços para desenvolver uma tecnologia que conecta objetos físicos capazes de trocar informações e perceber nuances do seu próprio ambiente, propiciando dados estratégicos para uma melhor tomada de decisão. Tal tecnologia é cunhada como Internet das Coisas, uma rede diferenciada estabelecida entre objetos heterogêneos para fornecer serviços e aplicações para inúmeras áreas de atuação. Esta rede possui características e funcionamento diferente das redes tradicionais, sendo uma potencial tecnologia para uso no processo de ensinoaprendizagem nas salas de aula do futuro. Este artigo destaca o uso desta rede no contexto educacional, identificando as potenciais aplicações que podem melhorar a eficiência do ensino e propiciar um maior engajamento e interesse dos alunos e professores.
\end{abstract}

\section{Introdução}

Atualmente, há uma forte demanda no setor educacional por tecnologias de informação e comunicação que auxiliem professores na tomada de decisão e que estabeleçam um processo de ensino-aprendizagem mais eficiente. Tecnologias como sistemas tutores inteligentes (STI), automação em salas de aulas (SmartClass), ambientes virtuais de 
VI Congresso Brasileiro de Informática na Educação (CBIE 2017)

Anais dos Workshops do VI Congresso Brasileiro de Informática na Educação (WCBIE 2017)

aprendizado (AVA), ferramentas colaborativas, e Internet das Coisas (do inglês Internet of Things - IoT), entre outros, expõem a necessidade de se ter ferramentas personalizadas para um aprendizado mais efetivo. Dentre estas tecnologias, IoT apresenta-se como uma das mais promissoras para conectar objetos de fabricantes distintos em uma rede comum [1]. Construir redes IoT em cenários educacionais proporciona vantagens importantes para um desempenho aprimorado do ensino-aprendizagem aplicado nas escolas e universidades ao redor do mundo. Em primeiro lugar, redes IoT permitem que objetos ou pessoas sejam rastreadas e localizadas em suas respectivas instituições, reduzindo esforço e tempo de atividades como a verificação da frequência do aluno, a localização de professores e o rastreio de objetos como laptops, livros, ou objetos do patrimônio institucional. Em segundo lugar, é possível, com a utilização de dispositivos da neurociência, identificar acuradamente os aspectos afetivos do aluno, auxiliando o professor a melhor adequar o conteúdo para um indivíduo ou para a sala como um todo. Assim, é possível monitorar a motivação e o engajamento dos alunos em uma dada tarefa ou disciplina, permitindo que estilos diferenciados de aprendizado sejam aplicados de acordo com as características destes estudantes. Por último, o uso de sistemas embarcados conectados na Internet propicia uma imersão sem precedentes, tornando o ambiente mais interativo e adequado às necessidades modernas de aprendizado.

Entretanto, a aplicação deste tipo de rede em contexto educacional enfrenta alguns desafios que requerem o desenvolvimento de pesquisas elaboradas para aumentar a eficiência destas redes. O primeiro desafio é estabelecer uma rede segura contra invasões. A falha de segurança nestas redes permitem ao invasor controlar o objeto físico, além de obter informações de identificação e localização desses objetos, acarretando sérios problemas de privacidade e de confidencialidade dos dados. O segundo desafio é a interoperabilidade de dispositivos e aplicações contidas nesta rede, já que fabricantes distintos definem seus próprios padrões proprietários, prejudicando a integração de todos os dispositivos desta rede. O terceiro desafio é o alto custo operacional e de implantação associado à infraestrutura de comunicação para a operação destas redes. O quarto desafio reside no gerenciamento inteligente de uma rede IoT, exigindo do sistema um forte poder de orquestração dos componentes IoT por meio de técnicas de aprendizado de máquina, sendo tal orquestração uma tarefa complexa.

Portanto, este artigo apresenta uma visão geral da tecnologia IoT e seus benefícios para a área de educação. O principal objetivo é descrever a tecnologia e seu estado da arte, identificando quais componentes e serviços são adequados para prover um aprendizado mais efetivo. Como objetivos específicos, define-se uma fundamentação dos principais conceitos utilizados na área e discute-se aspectos arquiteturais e de padronização. Concluise que aplicar IoT na educação é fundamental para que novas aplicações surjam nas salas 
VI Congresso Brasileiro de Informática na Educação (CBIE 2017)

Anais dos Workshops do VI Congresso Brasileiro de Informática na Educação (WCBIE 2017)

de aulas do futuro e permitam um ensino mais inovador, dinâmico e rico em dados e informações.

Este trabalho é organizado da seguinte maneira: A Seção 2 define a fundamentação teórica da tecnologia IoT, apresentando um resumo histórico, projeções de mercado, arquiteturas de rede, protocolos e padrões utilizados pela indústria para garantir a eficiência desta tecnologia. A Seção 3 detalha como a tecnologia IoT pode ser utilizada em cenários educacionais, enfatizando o potencial do uso de IoT nesses cenários e identificando as principais aplicações no processo de ensino-aprendizagem. A Seção 4 indica as tendências na área de IoT, apresentando o conceito de Internet de Tudo (IoE). Por último, as considerações finais deste trabalho são apresentadas.

\section{Internet das Coisas na Educação}

O termo Internet of Things foi cunhado originalmente em 1999 pelo professor Kevin Ashton do MIT ao descrever um sistema no qual objetos do mundo físico pudessem ser conectados à Internet através de sensores. Posteriormente, este professor escreveu um artigo denominado “esta coisa da Internet das coisas” [2], formalizando o conceito e os elementos que o envolvem. Desde então, tem-se observado um crescimento contínuo no interesse da academia e da indústria para desenvolver dispositivos interoperáveis que se comuniquem, percebem, processam, localizem, identifiquem e gerem dados e semântica apropriada em domínios restritos.

Um sistema IoT é definido como um paradigma de comunicação no qual dispositivos do mundo físico se integram em um ambiente e são capazes de serem identificados, localizados, conectados à Internet, realizarem processamento e semântica, além de gerarem dados sobre este ambiente e sobre a interação entre esses dispositivos [3]. Um dos principais fatores desta harmônica integração é o crescente aumento da capacidade de processamento dos dispositivos embarcados, a miniaturização e o barateamento dos mecanismos de comunicação como identificação por radiofrequência (RFID), rede de sensores, comunicação por campos próximos (NFC) e redes de comunicação sem fio, tornando o paradigma IoT cada vez mais aplicável a inúmeros cenários diferentes. Entre os possíveis cenários de aplicação deste paradigma, destaca-se o setor de transporte e logística, cuidados de saúde, ambientes inteligentes, aplicações de uso pessoal e educação.

No que concerne à implantação de soluções de IoT no cotidiano da sociedade, o crescimento desta área tem sido igualmente notável. Calcula-se que no ano de 2010 o número de objetos conectados na Internet tenha superado a população da terra e estima-se que em 2020 o número de objetos conectados alcance 212 bilhões de dispositivos, representando 45\% do tráfico da Internet [4][5]. Em termos de impacto financeiro, estimase que em 2025 o impacto econômico alcance 11,1 trilhões de dólares americanos [1][5]. 


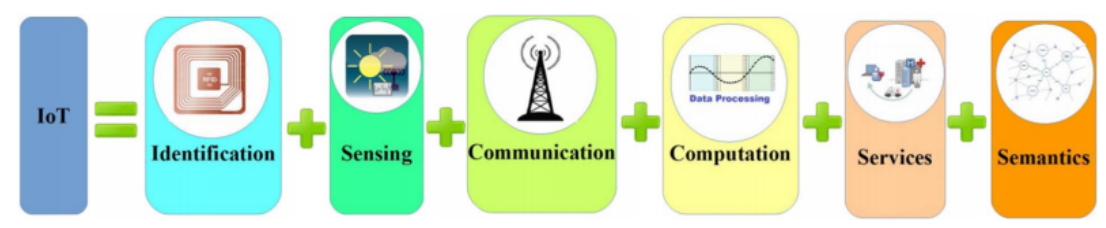

Fig 1. Principais elementos envolvidos no paradigma loT [3]

\subsection{Opções de Arquitetura para IoT na Educação}

A pesquisa na área de Internet das Coisas ainda não convergiu para um modelo único que represente uma arquitetura padrão para o desenvolvimento de uma solução nesta área [6] [7]. De modo geral, as propostas existentes na literatura propõem modelos em camadas semelhantes ao modelo de camadas da pilha TCP/IP (ainda que com funcionalidades diferentes em cada camada). Na Figura 2 são apresentadas algumas propostas de modelos arquiteturais. Como pode-se observar, as referidas propostas apresentam similaridades nas camadas, diferenciando-se no nível de detalhamento de cada proposta, onde as camadas inferiores tem como propósito básico a abstração do processo de comunicação dos objetos físicos e as camadas superiores são voltadas para o provimento de serviço propriamente dito.

Dentre as quatro opções de arquitetura existentes na Figura 2, a última opção, que inclui a camada de negócios, poderia controlar e conectar dispositivos IoT, sendo essencial para automação de salas que contenham processos mais abrangentes além do controle desses dispositivos. Por exemplo, a automação deverá integrar ambientes virtuais, sistemas tutores inteligentes, gerenciadores de projetos e controladores de ambientes, entre outros, para oferecer um ambiente de ensino-aprendizagem apropriado para alunos e professores. Utilizando este raciocínio, o uso de uma camada de negócios controlando a camada de aplicação é fundamental, pois permite que tais dispositivos IoT sejam controlados de acordo com a demanda destes processos.

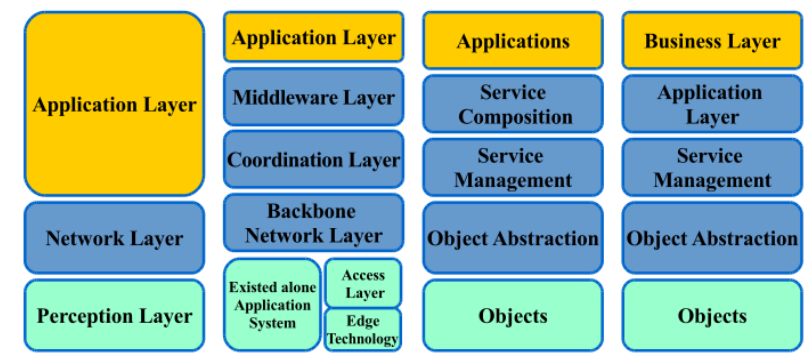

Figura 2: Propostas de modelos arquiteturais para loT. Figura extraída de [3].

\subsection{Protocolos e Padrões}


VI Congresso Brasileiro de Informática na Educação (CBIE 2017)

Anais dos Workshops do VI Congresso Brasileiro de Informática na Educação (WCBIE 2017)

Os protocolos tradicionais da Internet (HTTP, TCP, UDP, etc.) não foram projetados considerando a limitação dos dispositivos de IoT, tais como limitações de processamento e uso de bateria a ser empregada pelo dispositivo (hardware), faz-se necessário o desenvolvimento de novos protocolos ajustados para este ambiente de trabalho, buscando reduzir os conjuntos de mensagens para os dispositivos com pouca capacidade de processamento. Segundo [3][6][7], os principais protocolos utilizados no paradigma IoT são:

- Constrained Application Protocol ou CoAP, projetado para interagir com o protocolo HTTP, transportando mensagens e possuindo uma menor carga para os dispositivos móveis. Este protocolo apresenta as seguintes características: (i) UDP Nativo; (ii) segurança com DTLS (Datagram Transport Layer Security), em cima do UDP; (iii) Suporte Multicast; e (iv) comunicação assíncrona. Este protocolo é utilizado principalmente na automação residencial, sendo inserido no sistema operacional Contiki para controlar os dispositivos eletrônicos mais simples com suas memórias pequenas e com baterias de curta duração.

- Universal Plug and Play ou UPnP, projetado para realizar conexão dinâmica em dispositivos de computador. Este padrão utiliza um conjunto de protocolos da Internet e da WEB para permitir que dispositivos distintos se conectem em uma rede de forma automática, possibilitando a interoperabilidade entre PCs, periféricos, aparelhos inteligentes e dispositivos sem fio, sem a necessidade de qualquer configuração entre eles. Sua extensão é conhecida como UPnP+ e é compatível com IPv4 e IPv6, incorporando funcionalidades para conectividade de vários serviços disponibilizados pelos nós da rede.

- AllJoyn, um framework de código aberto que permite aos desenvolvedores criarem aplicações para que dispositivos possam encontrar e comunicar-se uns com os outros dentro de um espaço. Um consórcio de empresas de tecnologia como LG, Sharp, Cisco, TP-Link, Microsoft, entre outras, colaboram para manter este framework. O framework provê descoberta e controle para os dispositivos interligados em nuvem, sendo uma alternativa ao UPnP+. Ele também permite que dispositivos se comuniquem com outros ao seu redor, sendo usado com sensores de presença.

- Message Queuing Telemetry Transport ou MQTT, projetado para publicação e assinatura de mensagens. Os princípios da arquitetura deste protocolo foram criados para diminuir os requisitos de largura de banda da rede, garantir a entrega dos dados com alto grau de confiabilidade, permitindo que aplicações embarcadas sejam executadas, havendo um limite de processamento e recursos de memória. Sendo código aberto, permite que adaptações sejam feitas para projetos específicos. Sua 
VI Congresso Brasileiro de Informática na Educação (CBIE 2017)

Anais dos Workshops do VI Congresso Brasileiro de Informática na Educação (WCBIE 2017)

extensão é conhecida como Message Queuing Telemetry Transport for Sensor Networks (MQTT-SN) em que o principal objetivo era utilizar tecnologias de rádio freqüência como Bluetooth, Zigbee, RFID, entre outros.

\section{Potencial de IoT na Educação}

Existe uma crescente transformação no modelo padrão de ensino em diversos países, deixando de ser apenas um modelo baseado em aulas expositivas e se tornando um modelo de ensino mais heterogêneo, colaborativo, personalizado e engajador. Nos países pioneiros na implantação e manutenção deste modelo, nota-se que os alunos aprendem quando estão engajados para a resolução de um desafio ou problema passado em sala de aula. O aprendizado baseado em problema torna-se um modelo que explora melhor a experiência de cada usuário no processo de resolução de um determinado problema ou projeto. Neste caso, o uso de tecnologia tem se tornado primordial para um ensino multidisciplinar e alinhado com as demandas de mercado.

Os alunos estão expostos a uma elevada gama de informações devido principalmente ao uso da tecnologia Internet e suas aplicações WEB. Neste contexto, o desafio dos educadores é conduzir, de forma apropriada, o uso das tecnologias de informação e de comunicação para auxiliar neste processo educativo. Neste sentido, a tecnologia vem transformando o modo de aprender e de ensinar, exigindo dos professores métodos mais eficazes para o ensino. Um exemplo de método seria o processo de sala de aula invertida (i.e. flipped classroom), no qual aulas ou palestras podem ser assistidas diversas vezes e utilizadas como base para projetos práticos relacionados a uma lição específica. Isto é, as aulas presenciais são transformadas em sessões de um projeto e o foco do aprendizado está na realização deste projeto e não em assistir aulas expositivas.

Ambientes virtuais de aprendizagem propiciam aos alunos utilizarem blogs, fóruns, criar seu próprio conteúdo, compartilhar ou trocar ideias. Tais alunos conseguem respostas imediatas de um professor ou tutor e podem responder a questionários na própria ferramenta. O uso deste tipo de ferramenta permite ao estudante um aprendizado mais personalizado e coerente com o seu ritmo, possibilitando um ganho de desempenho maior neste aprendizado. Além disso, os professores conseguem acompanhar o rendimento do aluno, possibilitando ações customizadas e mais eficientes para que o aluno não perca a sua motivação ou o seu engajamento no processo de ensino-aprendizagem. Os professores podem compartilhar ou cooperar entre si por um conhecimento mais apurado e atual. Em suma, as salas de aula estão se tornando multimodais, com diversos recursos ou tecnologias para que o estudante não se desmotive e conclua seu aprendizado.

\subsection{Principais Aplicações}


VI Congresso Brasileiro de Informática na Educação (CBIE 2017)

Anais dos Workshops do VI Congresso Brasileiro de Informática na Educação (WCBIE 2017)

A tecnologia IoT aumenta a eficiência no processo de ensino-aprendizagem, quer seja automatizando tarefas cotidianas de uma sala de aula (e.g. presença automática de alunos em sala), quer seja em uma experiência de aprendizado mais personalizada (e.g. Etiquetas RFID, QR codes...), ou como um integrador de dados obtidos por sensores para tomada de decisões. Essas redes podem também capturar informações de contexto, cruzando, por exemplo, dados escolares do aluno com situações cotidianas (e.g. visita a um parque histórico, a uma igreja, ou outra localidade) para sugerir conteúdo ou alguma ação específica para o usuário. O aumento da eficiência operacional em qualquer ambiente educacional poderia ser feito através de uma rede de objetos físicos conectados na sala de aula, possibilitando que professores tenham o controle desses objetos e que possam realizar intervenções quando necessário, tornando assim uma aula mais dinâmica. Atualmente, existe uma solução de IoT chamada Twine7, consistindo em uma caixa que permite aos usuários ligar qualquer objeto físico a uma LAN. Este produto é de fácil configuração, integra sensores com serviços baseados na nuvem, apresentando informações pela WEB em tempo real. Qualquer indivíduo poderá receber informações do ambiente no qual a caixa está inserida.

\subsubsection{Interação em Aula}

Um dos maiores desafios entre os professores é descobrir quão motivadoras ou tediosas seriam as suas aulas. Neste caso, dispositivos vestíveis como roupas, pulseiras, bonés, etc, poderiam ser anexados aos alunos, rastreando padrões biológicos (e.g. uso de sensores ECG ou eletrocardiograma) para identificar picos coletivos de ansiedade, tédio, estresse ou desmotivação, ajudando o professor a tomar decisões.

\subsubsection{Ambiente Inteligente em Sala de Aula}

IoT pode ser utilizado em ambientes de sala de aula para aumentar a eficiência no processo de ensino-aprendizagem. Diversos objetos dentro destes ambientes podem ter dispositivos embarcados que trocam informações entre si, como por exemplo, lousas que interagem com dispositivos controlados pelo professor ou por alunos. Tais lousas podem estar conectadas com diversas universidades, ressaltando a colaboração entre instituições e tornando uma experiência mais enriquecedora para os alunos. Neste caso, estes alunos também podem interagir com outros alunos e profissionais conectados na Internet.

\subsubsection{Execução de Projetos}

Uma aula com o tema referente a características da fotossíntese poderia ser dada apresentando, além do conhecimento téorico, dados reais adquiridos por sensores espalhados em uma determinada floresta. Assim, dados empíricos reforçariam os conceitos dados em sala de aula.

\subsubsection{Eficiência Operacional}


VI Congresso Brasileiro de Informática na Educação (CBIE 2017)

Anais dos Workshops do VI Congresso Brasileiro de Informática na Educação (WCBIE 2017)

IoT pode ser utilizada para conservação de energia, transporte, ambiente, segurança, etc, reduzindo custos. Por exemplo, sensores, etiquetas RFID, câmeras e dispositivos conectados podem monitorar e vigiar um campus inteiro, notificando instantaneamente a uma central de polícia ou ao departamento de segurança da universidade.

\subsubsection{Estudantes especiais}

IoT pode auxiliar deficientes físicos. Por exemplo, sensores podem detectar usuários com algum tipo de deficiência e personalizar o conteúdo ou ações, como o tamanho de fonte, volume de som, chamar o professor para auxiliá-lo, entre outros.

\subsection{Fatores para implementação em instituições acadêmicas}

Para considerarmos a tecnologia IoT como uma rede essencial para um ensino mais acurado, é necessário que três pontos sejam bem definidos: (i) segurança; (ii) dispositivos embarcados; e (iii) políticas.

A segurança é um ponto fundamental para o sucesso de uma rede IoT. Quanto mais objetos estiverem conectados à uma rede pervasiva de ampla abrangência geográfica, maior deverá ser o esquema de segurança implementado para evitar acessos indevidos a dados pessoais. Neste caso, políticas de segurança devem ser implementadas para cada instituição envolvida, evitando assim quebras de privacidade dos seus dados na rede.

Dispositivos embarcados utilizados no IoT realizam comunicação e captura de dados do ambiente, colaborando, processando e enviando para usuários da rede informações deste ambiente, criando novas interações entre usuários e máquinas.

Políticas devem ser instaladas para estimular o uso da tecnologia em sala de aula e sua integração ao currículo acadêmico de uma instituição. Além disso, os professores devem oferecer novas metodologias para utilizar a rede IoT de forma completa e realizar mudanças no modelo pedagógico atual para transformar o ambiente atual de sala de aula.

\section{O futuro de IoT na Educação}

Em uma rede IoT, objetos físicos conversam entre si através de troca de mensagens para realizar alguma ação. Assim como as redes sociais na WEB se proliferam, é possível pensar em uma rede social entre máquinas, cooperando entre si para um determinado fim. Neste caso, em um cenário futuro, é possível que ambos objetos e pessoas se tornem uma grande rede social, viabilizando inúmeras aplicações para pesquisa e aprendizado. Segundo [1][5], o número de objetos físicos conectados à Internet ultrapassou a população da terra desde 2008, e cerca de 50 bilhões de objetos estarão conectados até 2020, o que ilustra a forte tendência no crescimento e no uso destes objetos.O mesmo trabalho prevê que IoE na educação terá um investimento de 175 bilhões de dólares nos próximos 10 anos para 
VI Congresso Brasileiro de Informática na Educação (CBIE 2017)

Anais dos Workshops do VI Congresso Brasileiro de Informática na Educação (WCBIE 2017)

desenvolver instruções personalizadas e capacidade de transmissão visando oferecer melhores tomadas de decisão e redução de gastos com recursos instrucionais.

A tecnologia IoT deverá se transformar em uma rede pervasiva global, sendo acessada, monitorada e controlada por toda a Internet. Neste cenário, os usuários estarão conectados às coisas (i.e. os dispositivos embarcados) que por sua vez estarão inseridas em um processo cujos dados serão elaborados para consumo das aplicações. Tal conceito mais abrangente é definido pela Cisco em [8] como IoE (Internet de Todas as Coisas ou Internet of Everything). Portanto, esta rede torna-se o próximo passo da conexão entre objetos físicos (i.e. IoT). A IoE enfatiza apenas comunicação entre máquinas utilizando rede de sensores e dados criados como resultado destas interações, transformando-se em uma rede que conecta bilhões de pessoas, processos dados e coisas. Tal integração torna conexões relevantes e essenciais para aplicações na área de educação, tornando a experiência mais rica e gerando diversas oportunidades de negócios.

\subsection{Pilares de IoE na Educação}

Segundo [8], quatro pilares sustentam o conceito IoE: (i) pessoas; (ii) processos; (iii) dados; e (iv) objetos. A seguir esses pilares são descritos e relacionados à educação.

\subsubsection{Pessoas}

Os provedores de acesso à Internet do século XXI tem presenciado um significativo aumento na adesão de pessoas aos seus serviços. Pessoas têm se conectado na rede mundial de computadores por diversos dispositivos (e.g. celulares, laptops, câmeras, dispositivos IoT) e utilizado redes sociais para manter suas conexões pessoais e profissionais. Para uma educação global, três

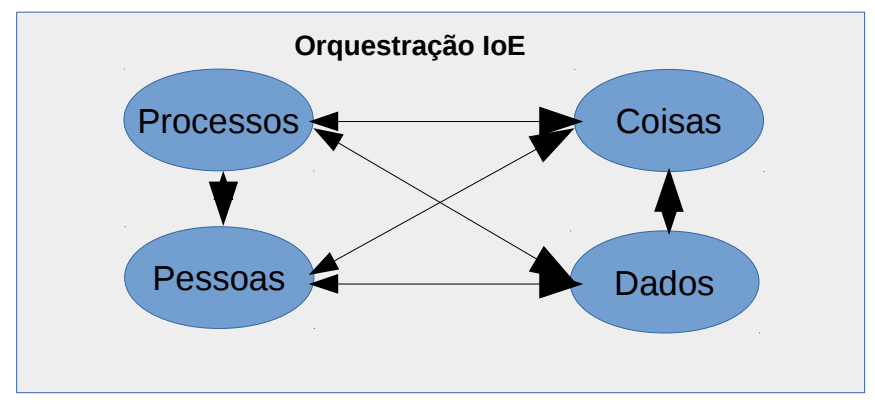

Figura 3: Orquestração loE.

passos são necessários: (i) personalização; (ii) treinamentos abertos e de alta qualidade; (iii) adoção de objetos inteligentes.

No primeiro passo, precisa-se entender o comportamento de cada usuário na Internet, direcionando o aprendizado de acordo com seu perfil. Encontrar o profissional certo para um dado problema, encontrar perfis similares para troca de informações, discutir 
VI Congresso Brasileiro de Informática na Educação (CBIE 2017)

Anais dos Workshops do VI Congresso Brasileiro de Informática na Educação (WCBIE 2017)

ideias, ou criar comunidades temáticas são exemplos de possíveis aplicações caso uma modelo do perfil de usuário seja definido e gerenciado por agentes inteligentes. No segundo passo, precisa-se implementar participação interativa em larga escala e acesso aberto para todos. Os cursos online abertos massivos (massive open online courses ou MOOCs) são exemplos de iniciativas que incentivam uma educação aberta e global, livre de taxas e cursos de alta qualidade permitindo a inclusão de inúmeros institutos que não teria condições de oferecer materiais de alta qualidade por restrições financeiras. Novas redes surgirão, conectando novas pessoas. No terceiro passo, objetos inteligentes obtém informações sobre custos, temperatura, umidade, calor, necessitando de trocas constantes de informação para que a rede se mantenha atualizada e preparada para eventuais riscos. Neste caso, IoT viabiliza o acesso aos dados destes objetos.

\subsubsection{Processo}

Processos definem como as pessoas, os dados e os objetos deverão interagir para gerar conhecimento utilizável. Estes processos permitem que a informação seja destinada a pessoa correta, no tempo certo e de forma apropriada. Como consequência, tal pessoa terá um maior oportunidade de aprendizado, tornando o processo educativo mais eficiente e motivador. Por exemplo, um aprendiz poderia verificar seu ranking em tempo real e comparar em relação aos seus concorrentes. O processo poderia eliminar exames que fizessem comparações entre desempenho. O feedback personalizado seria mais eficiente, permitindo que o aprendiz melhorasse sua compreensão acerca do conteúdo avaliado.

\subsubsection{Dados}

Dados são fundamentais para o sucesso do IoE. A tendência é que os dispositivos de IoT evoluam, alcançando um nível de inteligência que proporcionará dados mais relevantes sobre a rede, sobre as máquinas conectadas na rede, sobre os computadores e sobre as pessoas envolvidas na rede IoE, permitindo tomada de decisões mais acuradas. Por exemplo, estudantes poderiam anexar dispositivos IoT em objetos específicos, capturar dados referentes à estes objetos e repassar tais informações para programas que analisariam os detalhes minuciosamente. Estudantes também podem anexar dispositivos em animais e obter dados sobre seu habitat, seus comportamentos, seus movimentos, sua forma de caçar, etc. Podendo manipular dados de experimentos reais, os estudantes podem colaborar com o conteúdo em banco de dados, distribuindo dados para outros projetos de pesquisa, engajando os estudantes a colaborarem com outros pesquisadores em problemas locais ou globais. Isto faz com que a experi?ncia do usuário seja muito mais rica e forte no processo de ensino-aprendizagem, além de desenvolver a competência necessária para produzir e usar os dados em diversas situações locais. Dados sobre atividades diárias dos estudantes podem ser coletadas por dispositivos IoT, obtendo informações como a forma de caminhar, dados biológicos, funções metabólicas, sendo interessante para aulas de biologia, por 
VI Congresso Brasileiro de Informática na Educação (CBIE 2017)

Anais dos Workshops do VI Congresso Brasileiro de Informática na Educação (WCBIE 2017)

exemplo. IoE estimula a inovação e um aprendizado mais ativo por parte dos estudantes, ajudando os professores a terem informações mais precisas dos hábitos, da saúde e do impacto de suas aulas.

\subsubsection{Coisas}

Coisas são objetos físicos que se conectam na Internet e nas pessoas através de sensores. Tais sensores capturam dados que serão utilizados por aplicações cientes de contexto, ajudando as pessoas a terem informações mais precisas sobre o ambiente e sobre quais decisões obterão maiores impactos no processo de ensino-aprendizagem. Por exemplo, sensores podem ser utilizados em florestas para monitorar temperatura, pressão, umidade, luz, etc. Também podem ser utilizados para monitorar a estrutura de uma ponte, sua dilatação, vibração, etc. Estudantes obtêm tais dados por aplicativos instalados em seus computadores, aprendendo conceitos de física e de química. Sensores também podem ser utilizados para aprender o estilo de vida de diversos animais selvagens em um dado bioma terrestre. Por exemplo, o estilo de vida de um leão, seus hábitos, informações biológicas, velocidade, entre outros, pode ser utilizado para entender certos hábitos do animal, de forma que os estudantes pudessem comparar seus hábitos com animais da mesma classificação biológica e relatar cruzamentos, diferenças ou similaridades.

Na educação, sensores podem auxiliar crianças a se comunicarem corretamente na língua de sinais, por exemplo. O ensino dos gestos de LIBRAS pode ser feito por luvas com sensores, onde um programa avaliará se a movimentação e o tempo de movimentação está de acordo com um determinado padrão. Headsets também podem ser utilizados para identificar pessoas com déficit de atenção. Neste caso, sensores que detectam a atividade cerebral são instalados nestes headsets e um aplicação pode oferecer recompensas quando um estudante demonstra uma concentração melhorada em alguma atividade. $\mathrm{O}$ uso de sensores na educação ajuda os estudantes a compreenderem as consequências de seus atos previamente, pois eles podem identificar em tempo real seus comportamentos, ações e hábitos que afetam de alguma forma o ambiente. Este auto-estudo das ações implica em um contexto de aprendizado eficiente e divertido, com imenso potencial de engajamento e motivação desses estudantes.

\section{Considerações Finais}

Este artigo investigou o paradigma IoT através de suas aplicações, tendências e tecnologias envolvidas. O foco foi descrever a rede IoT como um conjunto de elementos capazes de permitir comunicação entre objetos, possibilitando que novas aplicações sejam criadas. Descreveu-se tais elementos, os protocolos e padrões envolvidos. Além disso, apresentouse as aplicações em potencial para o sucesso destas redes em ambientes futuros de salas de aula. Por último, descreveu-se o próximo passo evolutivo do IoT, integrando as coisas, 
VI Congresso Brasileiro de Informática na Educação (CBIE 2017)

Anais dos Workshops do VI Congresso Brasileiro de Informática na Educação (WCBIE 2017)

pessoas, dados e processos em um ecossistema auto-gerenciável. O desenvolvimento e a evolução de sistemas educacionais que utilizem a tecnologia IoT favorecerá o sistema tradicional de ensino, além de permitir que novas estratégias pedagógicas possam ser otimizadas em um ambiente de sala de aula. Dentre os inúmeros benefícios desta aplicação, destacam-se: (i) os estudantes aprenderão mais rápido; (ii) o processo educacional tornarse-á mais personalizado; (iii) sistemas educacionais com IoT propiciarão um forte incentivo a colaboração; (iv) Redes IoT permitem que os dispositivos sejam controlados eficazmente; (v) várias tarefas podem ser automatizadas, como frequência de alunos, avaliações, etc; e (vi) professores terão ferramentas adequadas para realizar suas aulas de forma eficiente.

Como trabalhos futuros, pretende-se investigar o desempenho das atuais arquiteturas deste tipo de rede em cenários de educação. Além disso, pretende-se desenvolver um framework que busque resolver problemas de interoperabilidade, segurança e orquestração dos objetos IoT.

\section{Referências}

[1] James, M. And Michael, C. And Peter, B. And Jonathan, W. And Richard, D. and Jacques, B. And Dan, A. (2015) "The Internet of Things: Mapping the Value Beyond the Hype." McKinsey Global Institute, Junho 2015.

[2] Ashton, K. (2009) That 'Internet of Things' thing. RFID Journal, 2009.

[3] Al-fuqaha, A. and Guizani, M. and Mohammadi, M. and Aledhari, M. and Ayyash, M. (2015). Internet of Things: A Survey on Enabling Technologies, Protocols, and Applications, in IEEE Communications Surveys \& Tutorials, vol. 17, no. 4, pages 23472376, IEEE.

[4] Khan, R.; Khan, S. U.; Zaheer, R.; Khan, S. "Future Internet: The Internet of Things architecture, possible applications and key challenges" in Proc. 10th Int. Conf. FIT, 2012, pages 257-260.

[5] Atzori, L. And Antonio, I. And Giacomo, M. (2010). The Internet of Things: A survey. Comput. Netw. Pages 2787-2805.

[6] Yang, Z. et al., (2011) "Study and application on the architecture and key technologies for IOT,” in Proc. ICMT, pages 747-751.

[7] Wu, M.; Lu, T. J.; Ling, F. Y.; Sun, J. ; Du, H. Y. (2010) "Research on the architecture of Internet of Things,” in Proc. 3rd ICACTE, 2010, pages V5-484-V5-487.

[8] Bradley, J. and Barbier, J. And Handler, D. (2013). "Embracing the Internet of Everything To Capture Your Share of \$14.4 Trillion”. White Paper, Cisco. 2013. 